\title{
Spanish authorities investigate how nurse contracted Ebola
}

\author{
Anne Gulland
}

London

Spanish authorities are investigating how a nurse contracted Ebola virus disease while caring for a patient in a Madrid hospital.

The nurse, who has not been named, was caring for two Spanish missionaries, Manuel Garcia Viejo and Miguel Pajares, who were flown home to Spain for treatment after contracting the disease in west Africa. Garcia Viejo died at the Carlos III Hospital on 25 September and Pajares died in August.

Authorities are investigating how a member of healthcare staff, who, the hospital insists, was wearing protective clothing, was able to contract the disease. The nurse's husband and another nurse have been put in quarantine, and public health authorities are tracing others who have been in contact with the infected patient.

The European Commission has also asked the Spanish authorities to explain how the nurse was infected, and a meeting of the commission's health security committee is due to discuss the matter. A spokesman for the commission said that it was a "priority" for the Spanish authorities to investigate what happened, as this is the first time that someone has contracted the disease outside the affected countries. The World Health Organization has also said that it is ready to provide support for Spain.

The nurse fell ill on 30 September and was admitted first to a hospital in Alcorón, south west of Madrid, before being transferred under police escort to the Carlos III Hospital. More than 50 people are being monitored at both hospitals.
The El Pais newspaper reported that the nurse was being treated with convalescent blood products—a treatment recommended for testing by WHO. The newspaper has also reported concerns from healthcare staff about their protective clothing.

Ben Neumann, lecturer in virology at the University of Reading, said that, although protective equipment can substantially reduce the risk of contracting the disease, "handling an Ebola patient will always involve some risk."

"Nurses face a problem in that a person who is sick with Ebola can make quite a lot of highly infectious waste, as the patient loses fluid through diarrhoea and vomiting. Those bodily fluids can contain millions of Ebola viruses, and it only takes one to transfer the infection," he said.

He added that the infection was sometimes spread when the health worker removed his or her protective clothing. "It is also possible that a tiny amount of Ebola-containing liquid splashed on the protective garments and then was transferred to her skin while removing the protective clothing," he said.

The latest figures from WHO showed 7470 cases of the disease in the three affected countries of Sierra Leone, Liberia, and Guinea, including 3431 deaths. In Nigeria there have been 20 cases with eight deaths, and there has been one case each in Senegal and the United States.

Cite this as: BMJ 2014;349:96120

๑ BMJ Publishing Group Ltd 2014 